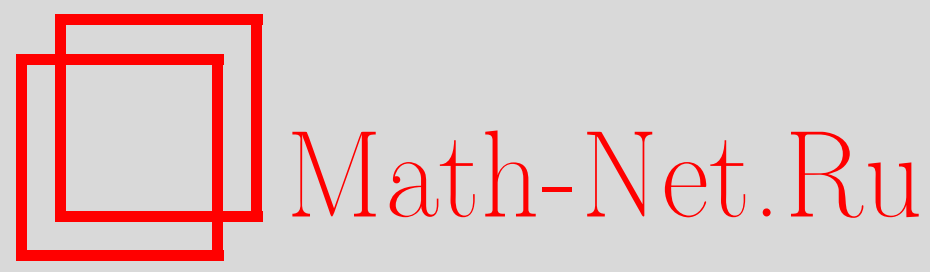

Г. В. Радзиевский, Теоремы существования обобщенных по Дзядыку моментных представлений, Матем. заметки, 2004, том 75, выпуск 2, 253-260

DOI: https://doi.org/10.4213/mzm31

Использование Общероссийского математического портала Math-Net.Ru подразумевает, что вы прочитали и согласны с пользовательским соглашением http://www.mathnet.ru/rus/agreement

Параметры загрузки:

IP : 54.198 .55 .26

26 апреля 2023 г., 13:04:43 


\section{ТЕОРЕМЫ СУЩЕСТВОВАНИЯ ОБОБЩЕННЫХ ПО ДЗЯДЫКУ МОМЕНТНЫХ ПРЕДСТАВЛЕНИЙ}

\section{Г.В. Радзиевский}

В работе, в частности, доказано, что для любой последовательности комплексных чисел $\left\{c_{n}\right\}_{n=0}^{\infty}$ найдется линейный замкнутый оператор $A$, действующий в гильбертовом пространстве, и два вектора $x$ и $y$, принадлежащие областям определения всех степеней оператора $A$, для которых $c_{n}=\left(A^{n} x, y\right)$. Если же ряд $\sum_{n=0}^{\infty} c_{n} z^{n}$ имеет радиус сходимости $R>0$, то в представлении $c_{n}=\left(A^{n} x, y\right)$ оператор $A$ можно выбрать ограниченным со спектральным радиусом, равным $1 / R$.

Библиографоял: 8 названий.

Исследования аппроксимаций Паде различных функций привело В. К. Дзядыка к понятию обобщенной проблемы моментов. Чтобы сформулировать эту задачу, введем необходимые обозначения. Везде далее (кроме замечания 2) $\mathfrak{H}$ - бесконечномерное гильбертово пространство над полем комплексных чисел $\mathbb{C}$ со скалярным произведением $(\cdot, \cdot)$, а $I$ - тождественньй в $\mathfrak{H}$ оператор. Пусть $\mathbb{N}$ и $\mathbb{N}_{0}-$ соответственно множества положительных и неотрицательных целых чисел.

Дзядыком в [1], [2; гл. VII, § 2] сформулирована следующая

ОБОБШЕННАЯ ПРОБЛЕМА МОМЕНТОВ. Пусть $\left\{c_{n}\right\}_{n \in \mathbb{N}}-$ произвольная последовательность комплексных чисел. Требуется найти гильбертово пространство $\mathfrak{H}$ и две такие последовательности векторов $\left\{a_{n}\right\}_{n \in \mathbb{N}_{0}} u\left\{b_{n}\right\}_{n \in \mathbb{N}_{0}}$ с әлементами $a_{n} u b_{n}$ из $\mathfrak{H}$, что

$$
c_{j+k}=\left(a_{j}, b_{k}\right), \quad j, k \in \mathbb{N}_{0} .
$$

Эти соотношения будем назьвать обобщенным по Дзядыку моментным представлением последовательности $\left\{c_{n}\right\}_{n \in \mathbb{N}_{0}}$.

Следует отметить, что в [1], [2] данная задача сформулирована в несколько более частном случае. А именно, в [1], [2] требовалось найти меру $\mu$ на множестве $X$, совпадающем либо с отрезком $[0 ; 1]$, либо с полуосью $[0 ; \infty)$, либо со всей осью $(-\infty ; \infty)$, и две такие последовательности функций $\left\{a_{n}\right\}_{n \in \mathbb{N}_{0}}$ и $\left\{b_{n}\right\}_{n \in \mathbb{N}_{0}}$ с элементами из $L_{2}(X, d \mu)$, чтобы выполнялись равенства (1). Кроме того, в [1], [2] присутствовало дополнительное условие о линейной независимости каждой конечной подпоследовательности у последовательностей функций $\left\{a_{n}\right\}_{n \in \mathbb{N}_{0}}$ и $\left\{b_{n}\right\}_{n \in \mathbb{N}_{0}}$. Это условие, как легко видеть, равносильно требованию отличия от нуля определителей Ганкеля $\operatorname{det}\left[c_{j+k}\right]_{j, k=0}^{n}$ при всех $n \in \mathbb{N}_{0}$.

Работаподдержана Государственньм фондом фундаментальных исследований Украины, проект Ф7/329-2001. 
При вьполнении этого требования сформулированная вьше задача была решена в [2, гл. VII, теорема 1], где (см. также [3], [4]) приведен ряд ее применений к аппроксимации Паде.

Заметим, что в связи с аппроксимацией Паде подобная задача, хотя и в неявном виде, возникала ранее (см., например, [5, часть $2, \S 2.3]$ и цитированную там литературу, а также [4]). А именно, требуется найти ограниченный линейный оператор $A$, действующий в гильбертовом пространстве $\mathfrak{H}$, и два таких вектора $x$ и $y$ из $\mathfrak{H}$, что

$$
c_{n}=\left(A^{n} x, y\right), \quad n \in \mathbb{N}_{0},
$$

причем здесь и далее $A^{0}:=I$. Если существует представление (2) последовательности $\left\{c_{n}\right\}_{n \in \mathbb{N}_{0}}$, то, полагая векторы $a_{j}:=A^{j} x$ и $b_{k}:=\left(A^{k}\right)^{*} y$ из (2), получаем (1). Следовательно, представление моментной последовательности $\left\{c_{n}\right\}_{n \in \mathbb{N}}$ в виде (2) является более общим нежели ее представление в виде (1). Счетная система равенств (2) допускает запись в виде одного равенства. Действительно, введем порождающую функцию

$$
f(z):=\sum_{n=0}^{\infty} c_{n} z^{n}
$$

последовательности $\left\{c_{n}\right\}_{n \in \mathbb{N}_{0}}$, и пусть $R_{A}(z):=(I-z A)^{-1}$. Тогда равенства (2) равносильны такому равенству:

$$
f(z)=\left(R_{A}(z) x, y\right) .
$$

Отсюда, в частности, заключаем, что требование ограниченности оператора $A$ в представлении (2) накладьвает весьма жесткое ограничение на последовательность $\left\{c_{n}\right\}_{n \in \mathbb{N}_{0}}$ : ее порождающая функция обязана быть голоморфной в окрестности нуля. При таком ограничении доказанная далее теорема 1 гарантирует сушествование представления (4), а значит, и (2). Если же отказаться от требования ограниченности оператора $A$, то, как следует из доказанных здесь теорем 1 и 2 , любую последовательность $\left\{c_{n}\right\}_{n \in \mathbb{N}_{0}}$ можно представить в виде (2), причем линейный оператор $A$ и векторы $x$ и $y$ выбраны так, что от представления (2) всегда можно перейти к представлению (1). Эти две теоремы, установленные в работе, дают полное решение более общей задачи, нежели задача о представлении обобщенной по Дзядыку моментной последовательности.

В работе неприведены приложения этих результатов к аппроксимации Паде, поскольку для этого пришлось бы просто повторить соответствующие рассуждения из [2]-[5].

Перейдем теперь к формулировкам и доказательствам основных результатов работы.

ТЕОремА 1. Пусть порождающая функиия $f$ последовательности $\left\{c_{n}\right\}_{n \in \mathbb{N}_{0}}$ голоморфна в окрестности нуля, а радиус сходимости ряда (3) равен $R(f)$. Тогда для любого бесконечномерного гильбертового пространства $\mathfrak{H}$ существует линейный ограниченный оператор $A$, действующий в $\mathfrak{H}$ и имеющий спектральный радиус $r(A)=1 / R(f)$, и әлементы $x, y \in \mathfrak{H}$, для которых справедливо представление (4).

ДокАЗАТЕЛЬСтво. Введем числа

$$
t_{n}:=\left(n^{2}\left|c_{n}\right|\right)^{1 / n}, \quad n \in \mathbb{N}
$$


где через $\alpha^{1 / n}$ обозначено арифметическое значение корня $n$-й степени из числа $\alpha \geqslant 0$. Согласно формуле Коши-Адамара $1 / R(f)=\lim _{\sup } \rightarrow \infty\left|c_{n}\right|^{1 / n}$, и поэтому

$$
\frac{1}{R(f)}=\limsup _{n \rightarrow \infty}\left|t_{n}\right| \text {. }
$$

Рассмотрим матрицы

$$
B_{n}:=\left[\delta_{j, s-1}\right]_{j, s=1}^{n+1}, \quad n \in \mathbb{N},
$$

где $\delta_{j, s}$ - символ Кронекера. Каждой матрице $B_{n}$ сопоставим линейный оператор $B_{n}$, действующий в комплексном $(n+1)$-мерном арифметическом унитарном пространстве $\mathbb{C}^{n+1}$ и имеющий в каноническом базисе $e_{l, n}=\left[\delta_{l, 1}, \ldots, \delta_{l, n+1}\right]^{T}, l=1, \ldots, n+1$, этого пространства матрицу $B_{n}$, причем систему векторов $e_{1, n}, \ldots, e_{n+1, n}$ считаем ортонормированной. Скалярное произведение в $\mathbb{C}^{n+1}$ обозначим через $(\cdot, \cdot)_{n}$.

Полагая $\operatorname{sign} \alpha:=\alpha /|\alpha|$ для неравного нулю комплексного числа $\alpha$ и $\operatorname{sign} 0:=0$, определим векторы

$$
\begin{gathered}
x_{1}:=\left[c_{0}, \operatorname{sign} c_{1}\right]^{T} \in \mathbb{C}^{2}, \quad x_{n}:=\left[0, \ldots, 0, \frac{\operatorname{sign} c_{n}}{n}\right]^{T} \in \mathbb{C}^{n+1}, \quad n=2,3, \ldots, \\
y_{n}:=\left[\frac{1}{n}, 0, \ldots, 0\right]^{T} \in \mathbb{C}^{n+1}, \quad n \in \mathbb{N} .
\end{gathered}
$$

Представим теперь гильбертово пространство $l_{2}$ в виде прямой суммы пространств $\mathbb{C}^{n+1}$, т.е.

$$
l_{2}=\bigoplus_{n=1}^{\infty} \mathbb{C}^{n+1}
$$

а скалярное произведение в $l_{2}$ обозначим через $(\cdot, \cdot)$.

И наконец, по введенным числам $t_{n}$, операторам $B_{n}$ и векторам $x_{n}$ и $y_{n}$ зададим оператор

$$
A:=\bigoplus_{n=1}^{\infty} t_{n} B_{n}
$$

действующий в пространстве $l_{2}$, и векторы

$$
x:=\bigoplus_{n=1}^{\infty} x_{n}, \quad y:=\bigoplus_{n=1}^{\infty} y_{n}
$$

принадлежащие $l_{2}$, и покажем, что для них справедливо представление (4).

Пусть $\theta_{n, n}-$ нулевой оператор в $\mathbb{C}^{n+1}$. Поскольку матрица оператора $B_{n}$ в ортонормированном базисе пространства $\mathbb{C}^{n+1}$ имеет вид (7), то $B_{n}^{k}=\theta_{n, n}$ при $k>n$, а $\left\|B_{n}^{k}\right\|=1$ при $1 \leqslant k \leqslant n$. Значит,

$$
A^{k}=\left(\bigoplus_{n=1}^{k-1} \theta_{n, n}\right) \oplus\left(\bigoplus_{n=k}^{\infty} t_{n}^{k} B_{n}^{k}\right), \quad k \in \mathbb{N}
$$

причем здесь и далее в аналогичньх формулах при $k=1$ левая часть равенства совпадает со вторым слагаемьм из правой. Тем самым, $\left\|A^{k}\right\|=\sup _{n \in \mathbb{N}: n \geqslant k}\left|t_{n}\right|^{k}$. Отсюда, 
принимая во внимание формулу Гельфанда для вычисления спектрального радиуса оператора и равенство (6), имеем

$$
r(A)=\lim _{k \rightarrow \infty}\left\|A^{k}\right\|^{1 / k}=\limsup _{n \rightarrow \infty}\left|t_{n}\right|=\frac{1}{R(f)} .
$$

Как уже отмечалось, $B_{n}^{k}=\theta_{n, n}$ при $k>n$. Следовательно,

$$
R_{t_{n} B_{n}}(z)=\sum_{k=0}^{n} t_{n}^{k} B_{n}^{k} z^{k}, \quad n \in \mathbb{N},
$$

при этом $t_{n}^{0}:=0$, если $t_{n}=0$. Из вида (7) матрищ операторов $B_{n}$ и определений (8) и $(9)$ векторов $x_{n}$ и $y_{n}$ вытекают соотношения $\left(B_{n}^{k} x_{n}, y_{n}\right)_{n}=\left(\delta_{k, n} \operatorname{sign} c_{n}\right) / n^{2}$ при $k, n \in \mathbb{N}$ и $\left(x_{1}, y_{1}\right)_{1}=c_{0}$, из которых с учетом определения (5) чисел $t_{n}$ и равенств (13) получаем, что

$$
\left(R_{t_{1} B_{1}}(z) x_{1}, y_{1}\right)_{1}=c_{0}+c_{1} z, \quad\left(R_{t_{n} B_{n}}(z) x_{n}, y_{n}\right)_{n}=c_{n} z^{n}, \quad n=2,3, \ldots
$$

Но так как

$$
\left(R_{A}(z) x, y\right)=\sum_{n=1}^{\infty}\left(R_{t_{n} B_{n}}(z) x_{n}, y_{n}\right)_{n},
$$

то отсюда следует требуемое представление (4). То, что спектральньй радиус оператора $A$ связан с радиусом сходимости ряда (3) формулой $r(A)=1 / R(f)$, было показано выше в (12).

Итак, теорема 1 доказана в случае, когда гильбертово пространство $\mathfrak{H}$ совпадает $c l_{2}$. Но поскольку все бесконечномерные сепарабельные гильбертовыпространства изометричны, то указанная конструкция дает необходимое представление в произвольном бесконечномерном сепарабельном гильбертовом пространстве $\mathfrak{H}$. Если же $\mathfrak{H}$ - несепарабельное гильбертово пространство, то оно допускает представление $\mathfrak{H}=\mathfrak{H}_{1} \oplus \mathfrak{H}_{2}$, где $\mathfrak{H}_{1}$ - бесконечномерное сепарабельное, а $\mathfrak{H}_{2}$ - несепарабельное гильбертовы пространства. Применяя доказанное утверждение теоремы 1 для пространства $\mathfrak{H}_{1}$, получаем $f(z)=\left(R_{A_{1}}(z) x_{1}, y_{1}\right)$, где оператор $A_{1}$ действует в $\mathfrak{H}_{1}$ и $r\left(A_{1}\right)=1 / R(f)$, а $x_{1}$ и $y_{1}$ - некоторые векторы из $\mathfrak{H}_{1}$. Далее, полагая $A=A_{1} \oplus \theta$ (здесь $\theta$ - нулевой оператор, действующий в $\left.\mathfrak{H}_{2}\right)$, а $x=x_{1} \oplus \theta$ и $y=y_{1} \oplus \theta$ (здесь уже $\theta$ - нулевой вектор из $\left.\mathfrak{H}_{2}\right)$, находим нужное представление в несепарабельном гильбертовом пространстве $\mathfrak{H}$. Тем самым теорема 1 полностью доказана.

СЛЕДСТВИЕ. Пусть выполнены условия теоремы 1. Тогда для любого числа $r \in[1 / R(f) ; \infty)$ и произвольного бесконечномерного гильбертова пространства $\mathfrak{H}$ существует линейный ограниченный оператор $A$, действующий в $\mathfrak{H}$ и имеющий спектральный радиус $r(A)=r$, и әлементы $x, y \in \mathfrak{H}$, для которых справедливо представление (4).

ДокАЗАТЕЛЬСТвО. Представим гильбертово пространство $\mathfrak{H}$ в виде суммы $\mathfrak{H}=\mathfrak{H}_{1} \oplus$ $\mathfrak{H}_{2}$, где $\mathfrak{H}_{2}-$ какое-либо одномерное подпространство пространства $\mathfrak{H}$. Применяя теорему 1 , находим оператор $A_{1}$ c $r\left(A_{1}\right)=1 / R(f)$, действующий в $\mathfrak{H}_{1}$, и векторы $x_{1}, y_{1} \in \mathfrak{H}_{1}$, для которых $f(z)=\left(R_{A_{1}}(z) x_{1}, y_{1}\right)$. Далее, введем оператор $A_{2}$, действующий в $\mathfrak{H}_{2}$ по правилу: $A_{2} x=r x, x \in \mathfrak{H}_{2}$, и рассмотрим в $\mathfrak{H}$ оператор $A=A_{1} \oplus A_{2}$ и векторы $x=x_{1} \oplus \theta$ и $y=y_{1} \oplus \theta$ (здесь $\theta-$ нулевой вектор пространства $\left.\mathfrak{H}_{2}\right)$. Тогда $r(A)=r, \mathrm{a}$ $\left(R_{A}(z) x, y\right)=f(z)$. 
ЗАмечАние 1 . Пусть $D_{R}:=\{z \in \mathbb{C}:|z|<R\}-$ круг конечного радиуса $R>0$ с центром в нуле, $\partial D_{R}$ - его гранища, а $0<p \leqslant \infty$. Обозначим через $H_{p}\left(D_{R}\right)$ пространство Харди голоморфных в круге $D_{R}$ функций (см., например, [6]), а через $C\left(\partial D_{R}\right)-$ пространство непрерывных на окружности $\partial D_{R}$ функций с нормой максимума модуля функции на $\partial D_{R}$. Если $f \in H_{1}\left(D_{R}\right)$, то утверждение теоремы 1 с заменой в нем произвольного гильбертового пространства $\mathfrak{H}$ на банахово пространство $C\left(\partial D_{R}\right)$ можно извлечь из [7; гл 2, теорема 2.4]. При этом равенство (4) запишется в виде $f(z)=y\left(R_{A}(z) x\right)$, где $y$ - некоторый линейньй непрерьвньй функционал на $C\left(\partial D_{R}\right)$. Если же еще усилить требование на $f$ и считать, что $f \in H_{2}\left(D_{R}\right)$, то из конструкции, имеющейся в [7] и в заключительной части доказательства теоремы 1 , можно получить утверждение теоремы 1 в произвольном бесконечномерном гильбертовом пространстве.

Как уже отмечалось, утверждение (4) теоремы 1 допускает равносильную запись в виде (2). Оказывается, что в такой формулировке теорему 1 удается уточнить, заменив в ней голоморфную в окрестности нуля функцию произвольным степенным рядом, имеющим нулевой радиус сходимости. Естественно, что в этом случае представление (4), как и равносильное ему представление (2), с ограниченньм оператором невозможно, поскольку для ограниченного оператора $A$ функция $\left(R_{A}(\cdot) x, y\right)$ a priori будет голоморфной в круге $\{z \in \mathbb{C}:|z|<1 / r(A)\}$. (Хотя, как показано в следствии, функция $\left(R_{A}(\cdot) x, y\right)$ может допускать голоморфное продолжение и за пределы круга $\{z \in \mathbb{C}:|z|<1 / r(A)\}$ и, в частности, при $r(A)>0$ она может оказаться целой.) Однако, если отказаться от требования ограниченности оператора $A$, то представление $(2)$ удается построить для произвольной последовательности $\left\{c_{n}\right\}_{n \in \mathbb{N}_{0}}$.

Чтобы сформулировать соответствующее уточнение теоремы 1 , введем необходимые определения и обозначения.

Пусть $A$ - линейньй, вообще говоря, неограниченньй оператор, действующий в гильбертовом пространстве $\mathfrak{H}$ и имеющий область определения $\mathfrak{D}(A)$. Оператор $A^{k}$ определяется по индукции соотношениями: $A^{0}:=I, A^{1}:=A$, а при $k=2,3, \ldots$

$$
\mathfrak{D}\left(A^{k}\right):=\left\{f \in \mathfrak{D}\left(A^{k-1}\right): A^{k-1} f \in \mathfrak{D}(A)\right\}, A^{k} f:=A\left(A^{k-1} f\right), \quad f \in \mathfrak{D}\left(A^{k}\right) .
$$

Отсюда, в частности, следует, что $A^{j+k} f=A^{j}\left(A^{k} f\right)$ для всех векторов $f \in \mathfrak{D}\left(A^{j+k}\right)$ и индексов $j, k \in \mathbb{N}_{0}$. Обозначим через $\mathfrak{D}_{\infty}(A):=\bigcap_{k \in \mathbb{N}} \mathfrak{D}\left(A^{k}\right)$, и пусть $\widetilde{A^{k}}$ - замыкание оператора $A^{k}$ для $k \in \mathbb{N}$.

Во введенных обозначениях справедливо следующее утверждение.

ТЕОрема 2. Пусть $\left\{c_{n}\right\}_{n \in \mathbb{N}_{0}}$ - такая числовая последовательность, что степенной ряд $\sum_{n=0}^{\infty} c_{n} z^{n}$ имеет нулевой радиус сходимости, а $\mathfrak{H}-$ произвольное бесконечномерное гильбертово пространство. Тогда найдется такой линейный неограниченный замкнутый оператор $A$, действующий в $\mathfrak{H}$, что $\mathfrak{D}_{\infty}(A)$ всюду плотно в $\mathfrak{H}$, операторы $A^{k}$ при $k=2,3, \ldots$ допускают замыкание, множество

$$
\mathfrak{M}:=\mathfrak{D}_{\infty}(A) \cap \mathfrak{D}_{\infty}\left(A^{*}\right) \cap\left(\bigcap_{k \in \mathbb{N}} \mathfrak{D}\left(\left(A^{k}\right)^{*}\right)\right)
$$

всюду плотно в $\mathfrak{H}$, и найдутся векторы $x$ и у из $\mathfrak{M}$, для которых справедливь представления

$$
c_{j+k}=\left(A^{j+k} x, y\right)=\left(A^{j} x,\left(A^{k}\right)^{*} y\right)=\left(A^{j} x,\left(A^{*}\right)^{k} y\right), \quad j, k \in \mathbb{N}_{0} .
$$


ДокАЗАТЕЛЬСТво использует ряд построений, введенных при доказательстве теоремы 1 , поэтому далее без пояснений используются обозначения из этого доказательства. Здесь последовательность $\left\{t_{n}\right\}_{n \in \mathbb{N}}$ выбирается иной, нежели в (5), а именно, положим

$$
d_{n}:=\max \left\{1,\left|c_{n}\right|\right\}, \quad t_{n}:=\left(n^{2} d_{n}^{2}\left|c_{n}\right|\right)^{1 / n}, \quad n \in \mathbb{N},
$$

и введем оператор $A$, действующий в пространстве $l_{2}$, представленном в виде $(10)$, по правилу

$$
\begin{gathered}
\mathfrak{D}(A):=\left\{f=\bigoplus_{n=1}^{\infty} f_{n} \in l_{2}: f_{n} \in \mathbb{C}^{n+1}, \sum_{n=1}^{\infty}\left|t_{n}\right|^{2}\left\|B_{n} f_{n}\right\|^{2}<\infty\right\}, \\
A f:=\bigoplus_{n=1}^{\infty} t_{n} B_{n} f_{n}, \quad f \in \mathfrak{D}(A) .
\end{gathered}
$$

Считая $\theta_{n}$ нулевым элементом пространства $\mathbb{C}^{n+1}$, запишем согласно определению (14) степени оператора $A$ :

$$
\begin{aligned}
\mathfrak{D}\left(A^{k}\right)= & \left\{f=\bigoplus_{n=1}^{\infty} f_{n} \in l_{2}: f_{n} \in \mathbb{C}^{n+1},\right. \\
& \left.\sum_{n=j}^{\infty}\left|t_{n}^{j}\right|^{2}\left\|B_{n}^{j} f_{n}\right\|^{2}<\infty, \quad j=1, \ldots, k\right\}, \\
A^{k} f= & \left(\bigoplus_{n=1}^{k-1} \theta_{n, n}\right) \oplus\left(\bigoplus_{n=k}^{\infty} t_{n}^{k} B_{n}^{k} f_{n}\right), \quad f \in \mathfrak{D}\left(A^{k}\right), \quad k \in \mathbb{N} .
\end{aligned}
$$

Обозначим через $\mathfrak{L}$ линейное многообразие пространства $l_{2}$, состоящее из векторов $f=\bigoplus_{n=1}^{\infty} f_{n}$, для которых элементы $f_{n}$ принадлежат $\mathbb{C}^{n+1}$ и равны нулю для достаточно больших $n$. Очевидно, что $\mathfrak{L}$ всюду плотно в $l_{2}$ и $\mathfrak{L} \subset \mathfrak{D}\left(A^{k}\right)$ при всех $k \in \mathbb{N}$. Значит, $\mathfrak{D}_{\infty}(A)$ также всюду плотно в $l_{2}$ и у каждого оператора $A^{k}$ при $k \in \mathbb{N}$ имеется сопряженный. Вычислим эти сопряженные операторы. Пусть $B_{n}^{T}$ - транспонированная матрица к матрище $B_{n}$, заданной равенством (7), а $B_{n}^{T}$ - оператор, действующий в пространстве $\mathbb{C}^{n+1}$ и имеющий в каноническом базисе $e_{1, n}, \ldots, e_{n+1, n}$ этого пространства матрицу $B_{n}^{T}$. Согласно формуле (19), для любых векторов $f \in \mathfrak{D}\left(A^{k}\right)$ и $g=\bigoplus_{n=1}^{\infty} g_{n} \in l_{2}$ с $g_{n} \in \mathbb{C}^{n+1}$ справедливо равенство

$$
\left(A^{k} f, g\right)=\sum_{n=k}^{\infty} \sum_{s=1}^{n-k+1} t_{n}^{k}\left(f_{n}, e_{k+s, n}\right)_{n}\left(e_{s, n}, g_{n}\right)_{n} .
$$

Считая в этом равенстве $f$ произвольным элементом из $\mathfrak{L}$, заключаем, что уравнение $\left(A^{k} f, g\right)=(f, w)$ разрешимо относительно вектора $g$ тогда и только тогда, когда компоненты вектора $w=\bigoplus_{n=1}^{\infty} w_{n} \in l_{2}$ с $w_{n} \in \mathbb{C}^{n+1}$ представимы в виде $w_{n}=\bar{t}_{n}^{k} B_{n}^{T} g_{n}$, $n \in \mathbb{N}$. Теперь уже несложно получить представление оператора $\left(A^{k}\right)^{*}$ :

$$
\begin{aligned}
\mathfrak{D}\left(\left(A^{k}\right)^{*}\right) & =\left\{g=\bigoplus_{n=1}^{\infty} g_{n} \in l_{2}: g_{n} \in \mathbb{C}^{n+1}, \quad \sum_{n=k}^{\infty}\left|t_{n}^{k}\right|^{2}\left\|\left(B_{n}^{T}\right)^{k} g_{n}\right\|^{2}<\infty\right\}, \\
\left(A^{k}\right)^{*} g & =\left(\bigoplus_{n=1}^{k-1} \theta_{n, n}\right) \oplus\left(\bigoplus_{n=k}^{\infty} \bar{t}_{n}^{k}\left(B_{n}^{T}\right)^{k} g_{n}\right), \quad g \in \mathfrak{D}\left(\left(A^{k}\right)^{*}\right), \quad k \in \mathbb{N} .
\end{aligned}
$$


Аналогично показывается, что

$$
\begin{gathered}
\mathfrak{D}\left(\left(A^{k}\right)^{* *}\right)=\left\{f=\bigoplus_{n=1}^{\infty} f_{n} \in l_{2}: f_{n} \in \mathbb{C}^{n+1}, \sum_{n=k}^{\infty}\left|t_{n}^{k}\right|^{2}\left\|B_{n}^{k} f_{n}\right\|^{2}<\infty\right\}, \\
k \in \mathbb{N},
\end{gathered}
$$

и $\left(A^{k}\right)^{* *} f=A^{k} f$ для $f \in \mathfrak{D}\left(A^{k}\right)$.

Поскольку [8; гл. 3, теорема 5.29] $\left(A^{k}\right)^{* *}=\widetilde{A^{k}}$, то сравнивая области определений (18) и (22) операторов $A^{k}$ и $\left(A^{k}\right)^{* *}$, заключаем, что при $k=1$ оператор $A^{k}$ замкнут, а при $k=2,3, \ldots$ операторы $A^{k}$ не замкнуты. Кроме того, из равенств $(20)$ и $(21)$ при $k=1$ и определения (14) степени оператора имеем

$$
\mathfrak{D}\left(\left(A^{*}\right)^{k}\right)=\left\{g=\bigoplus_{n=1}^{\infty} g_{n} \in l_{2}: g_{n} \in \mathbb{C}^{n+1}, \sum_{n=j}^{\infty}\left|t_{n}^{j}\right|^{2}\left\|\left(B_{n}^{T}\right)^{j} g_{n}\right\|^{2}<\infty, j=1, \ldots, k\right\}
$$

и $\left(A^{*}\right)^{k} g=\left(A^{k}\right)^{*} g$ для $g \in \mathfrak{D}\left(\left(A^{k}\right)^{*}\right)$.

И, наконец, зададим векторы

$$
\begin{aligned}
x_{1}:=\left[d_{1} c_{0}, \frac{\operatorname{sign} c_{1}}{d_{1}}\right]^{T} \in \mathbb{C}^{2}, x_{n} & :=\left[0, \ldots, 0, \frac{\operatorname{sign} c_{n}}{n d_{n}}\right]^{T} \in \mathbb{C}^{n+1}, n=2,3, \ldots, \\
y_{n}: & :\left[\frac{1}{n d_{n}}, 0, \ldots, 0\right]^{T} \in \mathbb{C}^{n+1}, \quad n \in \mathbb{N},
\end{aligned}
$$

а по ним формулами (11) определим элементы $x$ и $y$ из $l_{2}$.

Принимая во внимание равенства (17)-(19), (24) и (11), заключаем, что

$$
\begin{aligned}
\left\|A^{k} x\right\|^{2} & =\sum_{n=k}^{\infty} \frac{\left|t_{n}^{k}\right|^{2}}{n^{2} d_{n}^{2}}=\sum_{n=k}^{\infty} \frac{\left(n^{2} d_{n}^{2}\left|c_{n}\right|\right)^{2 k / n}}{n^{2} d_{n}^{2}} \\
& \leqslant \sum_{n=k}^{\infty} \frac{\left(n^{2} d_{n}^{3}\right)^{2 k / n}}{n^{2} d_{n}^{2}}<\infty, \quad k \in \mathbb{N},
\end{aligned}
$$

поэтому $x \in \mathfrak{D}_{\infty}(A)$. Аналогично из равенств $(17),(20),(21),(23),(25)$ и (11) следует принадлежность вектора $y$ множествам $\mathfrak{D}_{\infty}\left(A^{*}\right)$ и $\bigcap_{k \in \mathbb{N}} \mathfrak{D}\left(\left(A^{k}\right)^{*}\right)$. Поскольку вектор $x$ принадлежит $\mathfrak{D}\left(A^{*}\right)$ и ядрам операторов $\left(A^{*}\right)^{2}$ и $\left(A^{k}\right)^{*}$ при всех $k \in$ $\mathbb{N}$, а вектор $y$ принадлежит ядру оператора $A$, то $x$ принадлежит множествам $\mathfrak{D}_{\infty}\left(A^{*}\right)$ и $\bigcap_{k \in \mathbb{N}} \mathfrak{D}_{\infty}\left(\left(A^{k}\right)^{*}\right)$, а $y$ - множеству $\mathfrak{D}_{\infty}(A)$. Значит, векторы $x$ и $y$ принадлежат множеству $\mathfrak{M}$, заданному формулой (15). Теперь утверждение (16) теоремы 2 , как и утверждение теоремы 1 , проверяется непосредственным вычислением векторов $A^{j+k} x, A^{j} x$ и $\left(A^{k}\right)^{*} y=\left(A^{*}\right)^{k} y$, с последующим вычислением скалярных произведений. Всюду плотность множества (15) в $l_{2}$ следует из вида $(18),(20)$ и (23) областей определения операторов $A^{k},\left(A^{k}\right)^{*}$ и $\left(A^{*}\right)^{k}$ и того очевидного факта, что всюду плотное в $l_{2}$ множество $\mathfrak{L}$ принадлежит областям определения этих операторов при всех $k \in \mathbb{N}$. 
Итак, теорема 2 доказанав случае, когда гильбертово пространство $\mathfrak{H}$ совпадает с $l_{2}$. Случай произвольного гильбертового пространства сводится к изученному точно так же, как это сделано в заключительной части доказательства теоремы 1. Следует также отметить, что поскольку сведение случая несепарабельного гильбертового пространства к случаю сепарабельного происходит за счет ортогонального добавления нулевого оператора, то при таком сведении всюду плотность множества (15) не изменится, так как к нему ортогонально добавляется все несепарабельное гильбертово пространство $\mathfrak{H}_{2}$.

ЗАмЕчАниЕ 2. Если в условиях теорем 1,2 и следствия все члены последовательности $\left\{c_{n}\right\}_{n \in \mathbb{N}_{0}}$ вещественны, то и все числа $t_{n}$, элементы матриц $B_{n}$ и координаты векторов $x_{n}$ и $y_{n}$ окажутся вещественными. Поэтому в этом случае утверждения теорем 1,2 и следствия справедливы в произвольном бесконечномерном гильбертовом пространстве $\mathfrak{H}$ над полем вещественных чисел. Это замечание еще раз подчеркивает различие приведенной в доказательстве теоремы 1 конструкции от построения из [7], которое по существу опирается на методы теории голоморфных векторнозначных функций.

\section{СПИСОК ЦИТИРОВАННОЙ ЛИТЕРАТУРЫ}

[1] Дзядык В. К. Об обобщении проблемы моментов // Докл. АН УкрССР. Сер. А, физ. -матем. и техн. наук. 1981. №6. С. 8-12.

[2] Дзядык В.К. Аппроксимационные методы решения дифференциальных и интегральных уравнений. Киев: Наукова думка, 1988.

[3] Дзядык В. К., Голуб А. П. Обобщенная проблема моментов и аппроксимации Паде. Препринт 81.58. Киев: АН УкрССР, Ин-т математики, 1981.

[4] Голуб А. П. Обобщенные моментные представления и рациональные аппроксимации. Препринт 87.25. Киев: АН УкрССР, Ин-т математики, 1987.

[5] Бейкер Дж., Грейвс-Моррис П. Аппроксимации Паде. М.: Мир, 1986.

[6] Гофман К. Банаховы пространства аналитических функций. М.: ИЛ, 1963.

[7] Bart H., Gohberg I., Kaashoek M. A. Minimal Factorization of Matrix and Operator Functions. Operator Theory: Advances and Applications. V. 1. Basel-Boston: Birkhäuser, 1979.

[8] Като Т. Теория возмущений линейных операторов. М.: Мир, 1972.

Институт математики НАН Украины 